\title{
Evaluation of three rapid diagnostic tests for the detection of human infections with Plasmodium knowlesi
}

Deshka Foster ${ }^{1,2}$, Janet Cox-Singh ${ }^{1,3}$, Dayang SA Mohamad ${ }^{1}$, Sanjeev Krishna ${ }^{1,4}$, Pek P Chin $^{5}$ and Balbir Singh ${ }^{1 *}$

\begin{abstract}
Background: Plasmodium knowlesi, a malaria parasite of Southeast Asian macaques, infects humans and can cause fatal malaria. It is difficult to diagnose by microscopy because of morphological similarity to Plasmodium malariae. Nested PCR assay is the most accurate method to distinguish $P$. knowlesi from other Plasmodium species but is not cost effective in resource-poor settings. Rapid diagnostic tests (RDTs) are recommended for settings where malaria is prevalent. In this study, the effectiveness of three RDTs in detecting P. knowlesi from fresh and frozen patient blood samples was evaluated.
\end{abstract}

Methods: Forty malaria patients (28 P. knowlesi, ten P. vivax and two P. falciparum) diagnosed by microscopy were recruited in Sarawak, Malaysian Borneo during a 16-month period. Patient blood samples were used to determine parasitaemia by microscopy, confirm the Plasmodium species present by PCR and evaluate three RDTs: OptiMAL-IT, BinaxNOW ${ }^{\circledR}$ Malaria and Paramax-3. The RDTs were also evaluated using frozen blood samples from 41 knowlesi malaria patients.

Results: OptiMAL-IT was the most sensitive RDT, with a sensitivity of $71 \%(20 / 28 ; 95 \% \mathrm{Cl}=54-88 \%)$ for fresh and 73\% (30/41; $95 \% \mathrm{Cl}=59-87 \%)$ for frozen knowlesi samples. However, it yielded predominantly falciparum-positive results due to cross-reactivity of the $P$. falciparum test reagent with $P$. knowlesi. BinaxNOW ${ }^{\circledR}$ Malaria correctly detected non-P. falciparum malaria in $P$. knowlesi samples but was the least sensitive, detecting only $29 \%(8 / 28 ; 95 \% \mathrm{Cl}=12-46 \%)$ of fresh and $24 \%(10 / 41 ; 95 \% \mathrm{Cl}=11-37 \%)$ of frozen samples. The Paramax-3 RDT tested positive for $P$. vivax with PCR-confirmed P. knowlesi samples with sensitivities of $40 \%(10 / 25 ; 95 \% \mathrm{Cl}=21-59 \%)$ with fresh and 32\% (13/41; $95 \% \mathrm{Cl}=17-46 \%)$ with frozen samples. All RDTs correctly identified P. falciparum- and P. vivax-positive controls with parasitaemias above 2,000 parasites/ $\mu$ l blood.

Conclusions: The RDTs detected Plasmodium in P. knowlesi-infected blood samples with poor sensitivity and specificity. Patients with $P$. knowlesi could be misdiagnosed as $P$. falciparum with OptiMAL-IT, P. vivax with Paramax-3 and more correctly as non-P. vivax/non-P. falciparum with BinaxNOW ${ }^{\circledR}$ Malaria. There is a need for a sensitive and specific RDT for malaria diagnosis in settings where $P$. knowlesi infections predominate.

Keywords: Plasmodium knowlesi, Malaria diagnostics, Rapid diagnostic tests

\section{Background}

Until recently only four types of Plasmodium (Plasmodium falciparum, Plasmodium vivax, Plasmodium malariae and Plasmodium ovale) were known to cause malaria in humans. However, a fifth species, Plasmodium knowlesi, has been identified as a cause of human malaria in almost

\footnotetext{
* Correspondence: bskhaira55@gmail.com

${ }^{1}$ Malaria Research Centre, Universiti Malaysia Sarawak, Kuching, Sarawak, Malaysia

Full list of author information is available at the end of the article
}

all countries in Southeast Asia (recently reviewed [1]) and extending to the Nicobar and Andaman Islands in India [2]. In Malaysian Borneo, P. knowlesi is the main cause of admissions for malaria in certain hospitals, including Sarikei Hospital, and can lead to fatal infections [3-9].

Plasmodium species infections are typically diagnosed by microscopic examination of stained blood films, but there are limitations in sensitivity and specificity [10]. Nested PCR assays were developed to accurately distinguish 
between Plasmodium species. Molecular methods are the most sensitive detection method for malaria and can distinguish $P$. knowlesi from the morphologically similar $P$. malariae $[11,12]$. However, both microscopy and especially nested PCR assays require significant resources, such as specialized equipment, electricity and skilled technicians. PCR methods are not recommended for malaria diagnosis in resource-poor settings.

Malaria antigen-based rapid diagnostic tests (RDTs), mainly for the detection of $P$. falciparum, were developed for use in remote areas with limited facilities. A large number of RDTs are available for malaria diagnosis, including OptiMAL-IT produced by DiaMed, CA, USA $[13,14]$, BinaxNOW ${ }^{\oplus}$ Malaria produced by Inverness Medical, FL, USA [15,16], and Paramax-3 malaria Pf/ Pv/Pan from Zephyr Biomedical Systems, India $[17,18]$. The OptiMAL-IT test strip contains the antibody 17E4, which specifically detects $P$. falciparum lactate dehydrogenase (pLDH) and a pan-malaria antibody (19G4) that identifies $\mathrm{pLDH}$ from $P$. vivax, $P$. malariae and $P$. ovale as well as $P$. falciparum. BinaxNOW ${ }^{\oplus}$ Malaria is the only test approved by the US FDA and contains an antibody that detects $P$. falciparum-specific histidine rich protein 2 (HRP-2) and a pan-malaria antibody that detects Plasmodium aldolase, a glycolytic enzyme produced by all species of the parasite. The Paramax-3 test strip contains three antibody test lines as well as a control line: an antibody detecting P. falciparum HRP-2, similar to the BinaxNOW ${ }^{\bullet}$ Malaria test, a pan-malaria $\mathrm{pLDH}$ antibody, and an antibody specific for $P$. vivax LDH. None of the tests include P. knowlesi-specific reagents.

OptiMAL-IT, and another RDT under development [19], have been shown to detect $P$. knowlesi under experimental conditions. An imported case of human P. knowlesi from Sarawak to the Netherlands with $2 \%$ parasitaemia $(84,000$ trophozoites/ $\mu \mathrm{l})$ gave positive results for both the $P$. falciparum-specific LDH and pan-malarial LDH test lines, suggesting cross-reactivity between $P$. knowlesi LDH and the monoclonal antibody used in the test to detect P. falciparum LDH [20]. This cross-reactivity was first reported by McCutchan et al. [19], was also noted by Kawai et al. using P. knowlesi-infected Japanese macaque blood samples with OptiMAL-IT [21] and by Ong et al. using a $P$. knowlesi-positive blood sample from a patient with $0.2 \%$ parasitaemia [22].

BinaxNOW ${ }^{\oplus}$ Malaria has been used to detect $P$. knowlesi in several case reports with varying results [20,22-25]. In a case of $P$. knowlesi malaria imported into the Netherlands, BinaxNOW ${ }^{\oplus}$ Malaria but not OptiMAL-IT gave a positive result for non-P. falciparum malaria [20]. Similar results were reported in another case [23]. However, BinaxNOW ${ }^{\bullet}$ can also cross react and show falciparum-positive results, as observed with a $P$. knowlesi-infected patient with $0.2 \%$ parasitaemia [22]. False-negative results were also obtained using this test with two P. knowlesi malaria patients with parasitaemia of 0.0005 and $0.1 \%$ [24,25], suggesting that BinaxNOW ${ }^{\circ}$ Malaria may be less sensitive than OptiMAL-IT.

None of the aformentioned studies has evaluated the Paramax-3 test with P. knowlesi samples. The objective of this study was to evaluate three RDTs, OptiMAL-IT, BinaxNOW ${ }^{\oplus}$ Malaria, and Paramax-3, in the detection of P. knowlesi infection from both fresh and frozen blood samples from knowlesi malaria patients.

\section{Methods}

\section{Study sites}

All consecutive malaria-positive patients, diagnosed by microscopy, were recruited following acquisition of informed consent at Sarikei Polyclinic and Sarikei Hospital in the town of Sarikei, Sarawak, Malaysian Borneo. The study was approved by the Malaysian Ministry of Health's Medical Research and Ethics Committee.

Collection and processing of fresh and frozen blood samples A total of 40 malaria-positive blood samples were collected between March 2010 and July 2011: ten (seven P. knowlesi and three P. vivax) from Sarikei Polyclinic and 30 (21 P. knowlesi, seven P. vivax and two P. falciparum) from Sarikei Hospital. No mixed species infections were identified. Venous blood samples collected from these patients were used to make thick and thin blood films for verification of parasitaemia by microscopy, blood spots on filter paper for malaria species identification by nested PCR assays, and to evaluate RDTs.

The three RDTs were also evaluated using 44 frozen whole-blood samples collected from malaria patients with PCR-confirmed $P$. knowlesi $(\mathrm{N}=41)$, P. falciparum $(\mathrm{N}=3)$ and $P$. vivax $(\mathrm{N}=1)$, admitted to Sarikei and Sibu Hospitals in the two years prior to the start of this study and from those recruited during a previous study at Kapit Hospital [26]. Whole-blood samples, stored at $-80^{\circ} \mathrm{C}$, were thawed and used to evaluate the RDTs as recommended.

\section{Analysis of samples by microscopy}

Thick blood films from samples acquired at Sarikei Polyclinic and Hospital were allowed to dry overnight, then stained with 3\% Giemsa for 45 minutes. Thin blood films were fixed with methanol and stained with $10 \%$ Giemsa for 30 minutes. Parasitaemia was later determined by an experienced microscopist. Parasitaemia was calculated as the number of parasites per $\mu \mathrm{l}$ of blood for each sample by using each patient's actual white blood cell (WBC) count, and by counting up to $500 \mathrm{WBCs}$ in thick blood films and the corresponding number of malaria parasites. 


\section{Analysis of samples by nested PCR assay}

DNA was extracted from the blood spots on filter paper using the Instagene method [26]. All samples were initially examined by PCR assay using the Plasmodium genus-specific primers rPLU1 and rPLU5 for the Nest 1 amplification, which targets the small sub-unit ribosomal RNA gene of the Plasmodium genus [21]. Reaction mixtures for no more than ten samples were prepared and processed at one time and a positive and negative control were processed with each batch of samples to maintain high-quality control.

In order to identify the malaria species present in each sample, Nest 1 amplification products were screened using Nest 2 species-specific primers, as described previously $[12,26,27]$ for each of the five species of malaria known to infect humans: P. falciparum, P. vivax, P. malariae, $P$. ovale and P. knowlesi. For the species-specific primer pairs for $P$. falciparum, $P$. vivax, $P$. malariae and $P$. ovale (rFAL1/rFAL2, rVIV1/rVIV2, rMAL1/rMAL2, and rOVA1/rOVA4) the annealing temperature was $58^{\circ} \mathrm{C}$, and for the P. knowlesi-specific primer pair (kn1f and $\mathrm{kn} 3 \mathrm{r}$ ) the annealing temperature was $62^{\circ} \mathrm{C}$. The Nest 2 products were analysed using agarose gel (2.5\%) electrophoresis and Sybr green ${ }^{\circledast}$ staining (1x concentration for 30 minutes), viewed via UV transilluminator, photographed for documentation and recorded.

\section{Analysis of samples by RDTs}

The three antigen-based RDTs, OptiMAL-IT, BinaxNOW ${ }^{\circ}$ Malaria, and Paramax-3, were used according to the manufacturers' instructions. Fresh blood samples from malaria patients were processed within three hours of collection, and immediately when using thawed frozen blood samples. Thawed samples were processed as for fresh blood. OptiMAL-IT requires $10 \mu \mathrm{l}$ of blood and takes 20 minutes to complete, BinaxNOW ${ }^{\odot}$ Malaria requires $15 \mu \mathrm{l}$ of blood and takes 10-15 minutes to complete, and Paramax-3 requires $5 \mu$ l of blood and takes 15 minutes to complete. Tests were interpreted as successful when the control band was positive.

\section{Analysis of results of RDTs}

The sensitivity of each RDT was calculated with PCR results as the reference standard. The 95\% confidence interval (CI) for each test was calculated based on the test sensitivity and number of tests performed using 1.96 as the standard normal deviate (using the formula: $95 \%$ $\mathrm{CI}=$ Sensitivity $\pm 1.96 \times$ standard error of the test) [28].

\section{Results}

RDTs were evaluated against 40 microscopy-positive fresh blood samples (identified by nested PCR assay as 28 P. knowlesi, two P. falciparum and ten P. vivax), along with 44 frozen whole-blood malaria samples (41 P. knowlesi, two P. falciparum and one $P$. vivax by nested PCR assay). All RDTs were successful in that the control line was positive on all tests performed.

In total, 28 OptiMAL-IT and BinaxNOW ${ }^{\oplus}$ Malaria and 25 Paramax-3 tests were performed on P. knowlesi field isolates (median parasitaemia $=9,131$ parasites $/ \mu \mathrm{l}$; range $=159-911,616$ parasites $/ \mu$ l blood) and 41 of each RDT were performed using frozen $P$. knowlesi samples (median parasitaemia $=1,297$ parasites $/ \mu \mathrm{l}$; range $=10$ 188,384 parasites/ $\mu$ l blood).

OptiMAL-IT was the most sensitive of the three RDTs evaluated, with a sensitivity of $71 \%(20 / 28$; $95 \% \mathrm{CI}=54$ $88 \%)$ and $73 \%(30 / 41 ; 95 \% \mathrm{CI}=59-87 \%)$ for fresh and frozen knowlesi malaria samples, respectively (Tables 1 and 2). However, the test was not specific and P. knowlesi samples cross-reacted with the $P$. falciparum LDH test reagent in 18 of the 20 fresh samples identified. Only two of the fresh P. knowlesi samples were identified as non-P. falciparum malaria using OptiMAL-IT.

BinaxNOW ${ }^{\odot}$ Malaria showed the lowest sensitivity, detecting only $29 \%(8 / 28 ; 95 \% \mathrm{CI}=12-45 \%)$ of fresh and $24 \%(10 / 41 ; 95 \% \mathrm{CI}=11-37 \%)$ of frozen samples. This test was negative for all ten $P$. knowlesi fresh blood samples with parasitaemia $<5,000$ parasites/ $\mu$ l and also tested negative for $56 \%(10 / 18)$ of fresh samples with parasitaemia $>5,000$ parasites $/ \mu$ l (Table 1 ). However, with the BinaxNOW ${ }^{\oplus}$ Malaria test, which detects $P$. falciparumspecific histidine rich protein 2 (HRP-2), all positive results for $P$. knowlesi cases were correctly identified as non- $P$. falciparum malaria infections.

The Paramax-3 test also had low sensitivities of $40 \%$ (10/25; $95 \% \mathrm{CI}=21-59 \%)$ and 32\% (13/41; 95\% CI $=18$ 46\%) for fresh and frozen P. knowlesi samples, respectively. Of the ten Paramax-3 tests that yielded positive results with fresh blood samples, one indicated a $P$. falciparum infection, one indicated a non- $P$. falciparum, non- $P$. vivax result and the remaining eight gave results indicating $P$. vivax infections (Table 2 ).

Plasmodium vivax $(\mathrm{N}=10)$ and $P$. falciparum $(\mathrm{N}=2)$ samples from Sarikei Hospital and Polyclinic were collected for testing with RDTs as positive controls. All three RDTs gave positive results accurate for the species present when the parasitaemias were above 2,000 parasites/ $\mu$ l blood (range: 240-23,000 parasites/ $\mu \mathrm{l}$ ). The RDTs did not detect samples with $P$. vivax $(\mathrm{N}=2)$ or $P$. falciparum $(\mathrm{N}=1)$ parasitaemia less than 2,000 parasites/ $\mu$ l. All frozen $P$. vivax $(\mathrm{N}=1)$ and $P$. falciparum $(\mathrm{N}=2)$ positive controls yielded positive RDT results appropriate for the species. The parasitaemia for these samples was relatively high $(19,000$ 30,000 parasites/ $\mu \mathrm{l})$.

\section{Discussion}

Among the RDTs tested in this study, the OptiMAL-IT test was the most sensitive for P. knowlesi-infected blood 
Table 1 Rapid diagnostic test results for Plasmodium knowlesi fresh blood from field isolates

\begin{tabular}{|c|c|c|c|c|c|c|}
\hline \multirow{2}{*}{ 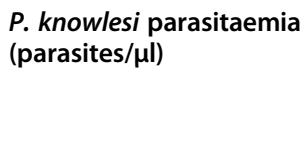 } & \multirow{2}{*}{ OptiMAL-IT } & \multirow[b]{2}{*}{$\begin{array}{l}\text { Number of } \\
\text { samples tested }\end{array}$} & \multicolumn{2}{|l|}{ BinaxNOW ${ }^{\circledR}$ Malaria } & \multirow[t]{2}{*}{ Paramax-3 } & \multirow[b]{2}{*}{$\begin{array}{l}\text { Number of } \\
\text { samples tested }\end{array}$} \\
\hline & & & & $\begin{array}{l}\text { Number of } \\
\text { samples tested }\end{array}$ & & \\
\hline \multirow[t]{4}{*}{$>5,000$} & $\begin{array}{l}\text { Pf-positive } \\
\text { (Pf and Pan-positive) }\end{array}$ & 13 & Pf-positive & 0 & $\begin{array}{l}\text { Pf-positive } \\
\text { (Pf and Pan-positive) }\end{array}$ & 1 \\
\hline & $\begin{array}{l}\text { Pan-positive } \\
\text { (Pv/Pm/Po) only }\end{array}$ & 1 & $\begin{array}{l}\text { Pan-positive } \\
\text { (Pv/Pm/Po) only }\end{array}$ & 8 & $\begin{array}{l}\text { Pv-positive } \\
\text { (Pv and Pan-positive) }\end{array}$ & 7 \\
\hline & & & & & $\begin{array}{l}\text { Pan-positive } \\
\text { (Pm/Po) only }\end{array}$ & 0 \\
\hline & $\underline{\text { Negative }}$ & 4 & Negative & 10 & Negative & 9 \\
\hline \multirow[t]{4}{*}{$1,001-5,000$} & $\begin{array}{l}\text { Pf-positive } \\
\text { (Pf and Pan-positive) }\end{array}$ & 4 & Pf-positive & 0 & $\begin{array}{l}\text { Pf-positive } \\
\text { (Pf and Pan-positive) }\end{array}$ & 0 \\
\hline & $\begin{array}{l}\text { Pan-positive } \\
\text { (Pv/Pm/Po) only }\end{array}$ & 1 & $\begin{array}{l}\text { Pan-positive } \\
\text { (Pv/Pm/Po) only }\end{array}$ & 0 & $\begin{array}{l}\text { Pv-positive } \\
\text { (Pv and Pan-positive) }\end{array}$ & 1 \\
\hline & & & & & $\begin{array}{l}\text { Pan-positive } \\
\text { (Pm/Po) only }\end{array}$ & 1 \\
\hline & Negative & 2 & Negative & 7 & Negative & 3 \\
\hline \multirow[t]{4}{*}{$501-1,000$} & $\begin{array}{l}\text { Pf-positive } \\
\text { (Pf and Pan-positive) }\end{array}$ & 0 & Pf-positive & 0 & $\begin{array}{l}\text { Pf-positive } \\
\text { (Pf and Pan-positive) }\end{array}$ & 0 \\
\hline & $\begin{array}{l}\text { Pan-positive } \\
\text { (Pv/Pm/Po) only }\end{array}$ & 0 & $\begin{array}{l}\text { Pan-positive } \\
\text { (Pv/Pm/Po) only }\end{array}$ & 0 & $\begin{array}{l}\text { Pv-positive } \\
\text { (Pv and Pan-positive) }\end{array}$ & 0 \\
\hline & & & & & $\begin{array}{l}\text { Pan-positive } \\
\text { (Pm/Po) only }\end{array}$ & 0 \\
\hline & $\underline{\text { Negative }}$ & 2 & Negative & 2 & Negative & 2 \\
\hline \multirow[t]{4}{*}{$1-500$} & $\begin{array}{l}\text { Pf-positive } \\
\text { (Pf and Pan-positive) }\end{array}$ & 1 & Pf-positive & 0 & $\begin{array}{l}\text { Pf-positive } \\
\text { (Pf and Pan-positive) }\end{array}$ & 0 \\
\hline & $\begin{array}{l}\text { Pan-positive } \\
\text { (Pv/Pm/Po) only }\end{array}$ & 0 & $\begin{array}{l}\text { Pan-positive (Pv/Pm/ } \\
\text { Po) only }\end{array}$ & 0 & $\begin{array}{l}\text { Pv-positive } \\
\text { (Pv and Pan-positive) }\end{array}$ & 0 \\
\hline & & & & & $\begin{array}{l}\text { Pan-positive } \\
\text { (Pm/Po) only }\end{array}$ & 0 \\
\hline & Negative & 0 & Negative & 1 & Negative & 1 \\
\hline \multirow[t]{4}{*}{ Total } & $\begin{array}{l}\text { Pf-positive } \\
\text { (Pf and Pan-positive) }\end{array}$ & 18 & Pf-positive & 0 & $\begin{array}{l}\text { Pf-positive } \\
\text { (Pf and Pan-positive) }\end{array}$ & 1 \\
\hline & $\begin{array}{l}\text { Pan-positive } \\
\text { (Pv/Pm/Po) only }\end{array}$ & 2 & $\begin{array}{l}\text { Pan-positive } \\
\text { (Pv/Pm/Po) only }\end{array}$ & 8 & $\begin{array}{l}\text { Pv-positive } \\
\text { (Pv and Pan-positive) }\end{array}$ & 8 \\
\hline & & & & & $\begin{array}{l}\text { Pan-positive } \\
\text { (Pm/Po) only }\end{array}$ & 1 \\
\hline & Negative & 8 & Negative & 20 & Negative & 15 \\
\hline Total tested & & 28 & & 28 & & 25 \\
\hline Total positive & & 20 & & 8 & & 10 \\
\hline Sensitivity & & $71 \%$ & & $29 \%$ & & $40 \%$ \\
\hline$(95 \% \mathrm{Cl})$ & & $(54-88 \%)$ & & $(12-46 \%)$ & & $(21-59 \%)$ \\
\hline
\end{tabular}

samples. However, the test was not specific and the majority of $P$. knowlesi samples were identified as $P$. falciparum by this test due to antibody cross-reactivity, as noted in previous studies [19-22]. BinaxNOW ${ }^{\bullet}$ Malaria was found to be the least sensitive of the three RDTs assessed, but there was no cross-reactivity observed between the $P$. falciparum antibody for $P$. falciparumHRP-2 and P. knowlesi samples. In this study, all positive results attained using this test with $P$. knowlesi-confirmed samples correctly indicated a non-P. falciparum malaria infection. The Paramax-3 test showed low sensitivity and cross-reactivity between the $P$. vivax LDH-detecting antibody and P. knowlesi. This observation has been noted in several other single case reports using different tests which also contain a $P$. vivax LDH-detecting antibody $[19,23,24]$.

The sensitivity of detecting P. knowlesi in blood samples with all three RDTs assessed in this study was significantly 
Table 2 Rapid diagnostic test results from frozen Plasmodium knowlesi blood samples

\begin{tabular}{|c|c|c|c|c|c|c|}
\hline \multirow{2}{*}{$\begin{array}{l}P . \text { knowlesi parasitaemia } \\
\text { (parasites/ } \mu \mathrm{l} \text { ) }\end{array}$} & \multirow{2}{*}{ OptiMAL-IT } & \multirow[b]{2}{*}{$\begin{array}{l}\text { Number of } \\
\text { samples tested }\end{array}$} & \multicolumn{2}{|l|}{ BinaxNOW ${ }^{\circledR}$ Malaria } & \multirow[t]{2}{*}{ Paramax-3 } & \multirow[b]{2}{*}{$\begin{array}{l}\text { Number of } \\
\text { samples tested }\end{array}$} \\
\hline & & & & $\begin{array}{l}\text { Number of } \\
\text { samples tested }\end{array}$ & & \\
\hline \multirow[t]{4}{*}{$>5,000$} & $\begin{array}{l}\text { Pf-positive } \\
\text { (Pf and Pan-positive) }\end{array}$ & 8 & Pf-positive & 0 & $\begin{array}{l}\text { Pf-positive } \\
\text { (Pf and Pan-positive) }\end{array}$ & 0 \\
\hline & $\begin{array}{l}\text { Pan-positive } \\
\text { (Pv/Pm/Po) only }\end{array}$ & 0 & $\begin{array}{l}\text { Pan-positive } \\
\text { (Pv/Pm/Po) only }\end{array}$ & 5 & $\begin{array}{l}\text { Pv-positive } \\
\text { (Pv and Pan-positive) }\end{array}$ & 7 \\
\hline & & & & & $\begin{array}{l}\text { Pan-positive } \\
\text { (Pm/Po) only }\end{array}$ & 0 \\
\hline & $\underline{\text { Negative }}$ & 0 & Negative & 3 & Negative & 1 \\
\hline \multirow[t]{4}{*}{$1,001-5,000$} & $\begin{array}{l}\text { Pf-positive } \\
\text { (Pf and Pan-positive) }\end{array}$ & 12 & Pf-positive & 0 & $\begin{array}{l}\text { Pf-positive } \\
\text { (Pf and Pan-positive) }\end{array}$ & 0 \\
\hline & $\begin{array}{l}\text { Pan-positive } \\
\text { (Pv/Pm/Po) only }\end{array}$ & 1 & $\begin{array}{l}\text { Pan-positive } \\
\text { (Pv/Pm/Po) only }\end{array}$ & 2 & $\begin{array}{l}\text { Pv-positive } \\
\text { (Pv and Pan-positive) }\end{array}$ & 4 \\
\hline & & & & & $\begin{array}{l}\text { Pan-positive } \\
\text { (Pm/Po) only }\end{array}$ & 0 \\
\hline & Negative & 0 & Negative & 11 & Negative & 9 \\
\hline \multirow[t]{4}{*}{$501-1,000$} & $\begin{array}{l}\text { Pf-positive } \\
\text { (Pf and Pan-positive) }\end{array}$ & 4 & Pf-positive & 0 & $\begin{array}{l}\text { Pf-positive } \\
\text { (Pf and Pan-positive) }\end{array}$ & 0 \\
\hline & $\begin{array}{l}\text { Pan-positive } \\
\text { (Pv/Pm/Po) only }\end{array}$ & 0 & $\begin{array}{l}\text { Pan-positive } \\
\text { (Pv/Pm/Po) only }\end{array}$ & 2 & $\begin{array}{l}\text { Pv-positive } \\
\text { (Pv and Pan-positive) }\end{array}$ & 1 \\
\hline & & & & & $\begin{array}{l}\text { Pan-positive } \\
\text { (Pm/Po) only }\end{array}$ & 0 \\
\hline & $\underline{\text { Negative }}$ & 4 & Negative & 6 & Negative & 7 \\
\hline \multirow[t]{4}{*}{$1-500$} & $\begin{array}{l}\text { Pf-positive } \\
\text { (Pf and Pan-positive) }\end{array}$ & 4 & Pf-positive & 0 & $\begin{array}{l}\text { Pf-positive } \\
\text { (Pf and Pan-positive) }\end{array}$ & 0 \\
\hline & $\begin{array}{l}\text { Pan-positive } \\
\text { (Pv/Pm/Po) only }\end{array}$ & 1 & $\begin{array}{l}\text { Pan-positive } \\
\text { (Pv/Pm/Po) only }\end{array}$ & 1 & $\begin{array}{l}\text { Pv-positive } \\
\text { (Pv and Pan-positive) }\end{array}$ & 1 \\
\hline & & & & & $\begin{array}{l}\text { Pan-positive (Pm/Po) } \\
\text { only }\end{array}$ & 0 \\
\hline & Negative & 7 & Negative & 11 & Negative & 11 \\
\hline \multirow[t]{4}{*}{ TOTAL } & $\begin{array}{l}\text { Pf-positive } \\
\text { (Pf and Pan-positive) }\end{array}$ & 28 & Pf-positive & 0 & $\begin{array}{l}\text { Pf-positive } \\
\text { (Pf and Pan-positive) }\end{array}$ & 0 \\
\hline & $\begin{array}{l}\text { Pan-positive } \\
\text { (Pv/Pm/Po) only }\end{array}$ & 2 & $\begin{array}{l}\text { Pan-positive (Pv/Pm/ } \\
\text { Po) only }\end{array}$ & 10 & $\begin{array}{l}\text { Pv-positive } \\
\text { (Pv and Pan-positive) }\end{array}$ & 13 \\
\hline & & & & & $\begin{array}{l}\text { Pan-positive } \\
\text { (Pm/Po) only }\end{array}$ & 0 \\
\hline & $\underline{\text { Negative }}$ & 11 & Negative & 31 & Negative & 28 \\
\hline Total tested & & 41 & & 41 & & 41 \\
\hline Total positive & & 30 & & 10 & & 13 \\
\hline Sensitivity & & $73 \%$ & & $24 \%$ & & $32 \%$ \\
\hline$(95 \% \mathrm{Cl})$ & & $(59-87 \%)$ & & $(11-37 \%)$ & & $(17-46 \%)$ \\
\hline
\end{tabular}

lower than that reported for other Plasmodium species. For example, the sensitivity of detection of $P$. falciparum using OptiMAL-IT has been reported as $95.3 \%$ (100\% for $>500$ parasites $/ \mu \mathrm{l}$ and $72 \%$ for 50 parasites $/ \mu \mathrm{l}$ ) and $96 \%$ for $P$. vivax malaria infections [14]. For BinaxNOW ${ }^{\circ}$ Malaria, the sensitivity of detection for $P$. falciparum has been reported as $95.3 \%(99.7 \%$ for $>5,000$ parasites/ $\mu \mathrm{l}$ and $53.9 \%$ for 100 parasites/ $\mu \mathrm{l}$ or fewer) and $68.9 \%$ for $P$. vivax malaria infections [16]. For Paramax-3, the sensitivity and specificity of detection for both $P$. falciparum and $P$. vivax malaria infections is reported as $100 \%$ in an in-house study of 251 samples [29]. Although the number of positive controls conducted in this study was relatively low, none of the RDTs used in this study detected $P$. vivax or $P$. falciparum infections in fresh blood samples with parasitaemias less than 2,000 parasites/ $\mu \mathrm{l}$.

One limitation of this study is the relatively low numbers of fresh samples tested. To strengthen the results 
from fresh samples, frozen blood samples were also included. The sensitivity of detection of knowlesi malaria infections with the RDTs tested were fairly similar using fresh versus frozen samples. Freeze-thawing and the storage of blood at low temperatures can accelerate deterioration of antigen activity, although it is also possible that target antigens are more accessible in freeze-thawed samples and may actually improve the sensitivity of RDTs [30].

A recently published paper from Sabah, Malaysian Borneo presents the use of two different RDTs, First Response $^{\mathrm{Ts}}$, which detects pan-Plasmodium LDH and Pf-specific HRP-2, and ParaHIT ${ }^{\mathrm{TM}}$, which detects panPlasmodium aldolase and Pf-specific HRP-2 [7]. A total of 129 P. knowlesi patient samples were studied, only 34 of whom were enrolled in the study prior to treatment, while the remainder were referred from district hospitals where they had already received anti-malarial treatment [7]. The findings of this study indicated a sensitivity of $74 \%$ for the pLDH component of the First Response ${ }^{\mathrm{Ts}}$ RDT, which is similar to that observed in the current study with OptiMAL-IT (71 and 73\% sensitivity with fresh and frozen samples, respectively), and higher than that observed with the Paramax-3 test (40 and 32\% sensitivity), both of which also detect $\mathrm{pLDH}$. In the current study as well as the one in Sabah, the RDTs with the pan-aldolase component had the lowest sensitivity of detection of P. knowlesi samples; $29 \%$ with the ParaHIT ${ }^{\mathrm{T \mu}}$ test [7], and 29\% with fresh and $24 \%$ with frozen samples using the BinaxNOW ${ }^{\oplus}$ Malaria RDT.

RDTs cost between 10 and 15 Malaysian Ringgit (US $\$ 3.17-4.80)$ per test when purchased at a dispensary in Malaysia. Although when purchased in bulk for malaria control programs this cost tends to be significantly reduced, microscopy is still the most affordable diagnostic tool and costs just one Ringgit (US\$0.30) per patient to screen for malaria. The cost of nested PCR assay is comparable to RDTs per patient sample, and although PCR assay is significantly more sensitive and specific than microscopy, this technique requires specialized equipment, electricity supply and training, and is not suitable for resource-poor settings. RDTs confer the advantages of speed (all types used in this study took 20 minutes or less to conduct), minimal training and ease of use, and do not require electricity or any specific hardware. However, currently available RDTs lack sensitivity and specificity compared to microscopy and PCR-based methods for all Plasmodium species infections, especially P. knowlesi. The development of loop-mediated isothermal amplification (LAMP) assays combine the sensitivity and specificity of PCR with low cost, low technology and rapid results. LAMP-based tests for malaria diagnosis that include reagents specific for $P$. knowlesi are under development and may be useful for resorce-poor settings [31,32].
In areas with relatively low malaria prevalence such as Sarawak, the cost of RDTs, even if sensitive and specific, would likely outweigh the benefit. To understand this in practical terms, consider, for example, the case of Julau Health Clinic, which is a small, rural health clinic in Sarawak surveyed as part of the current study. The prevalence of malaria at this clinic during a five-month study period in which 108 febrile patients whose clinical presentations were suggestive of malaria were screened using nested PCR assay was $0.2 \%$ (Foster et al., unpublished data). As such, it would have cost between MYR 1,080 and 1,620 (US\$342-832) to perform RDTs for these 108 query malaria patients and only two were positive.

This study confirms that the RDTs evaluated are not adequately sensitive for use in the diagnosis of $P$. knowlesi. Also, P. knowlesi cross-reacted with P. falciparum and $P$. vivax $\mathrm{LDH}$ antibodies used in two of the three commercially available RDTs tested, resulting in misdiagnosis of malaria species in an area where human P. knowlesi infections are prevalent. Since not all species of malaria warrant the same level of medical care, misidentification can result in mismanagement, especially when the potentially severe knowlesi malaria is misdiagnosed as vivax malaria. Because P. knowlesi is morphologically similar to P. malariae and P. falciparum, it is also misdiagnosed by microscopy [10,23,24]. However microscopy should not be replaced by RDTs in areas where P. knowlesi occurs until the sensitivity, specificity and costs are comparable.

\section{Conclusions}

The sensitivity of detection of $P$. knowlesi by the three RDTs evaluated is low compared with microscopy. Crossreactivity is common between $P$. knowlesi-infected blood and both the $P$. falciparum-detecting antibody used in the OptiMAL-IT test and the $P$. vivax-detecting antibody used in the Paramax-3 test. As such, a patient with knowlesi malaria may be diagnosed as $P$. falciparum by OptiMALIT, as $P$. vivax by Paramax-3 and as non-P.vivax and non-P. falciparum by BinaxNOW ${ }^{\odot}$ Malaria. Until more sensitive RDTs are developed that can distinguish $P$. knowlesi from P. falciparum and P. vivax, serious consideration should be taken before using RDTs for the diagnosis of malaria in settings where P. knowlesi is the predominant species.

\section{Competing interests}

The authors declare that they have no competing interests.

\section{Authors' contributions}

BS, JCS, SK and DF were involved in study conception and design,

procurement of RDTs and writing the manuscript. DF was involved in data collection and analysis, and drafting the manuscript. PPC assisted with data collection and analysis and DSAM assisted with laboratory data analysis. BS was involved in training of personnel and oversaw the project throughout. All the authors have read and approved the manuscript. 


\section{Acknowledgements}

We would like to thank the people who agreed to participate in this study in the Sarikei Division, and the medical directors, laboratory and nursing staff at the Sarikei Hospital and Polyclinic for their assistance with patient recruitment and sample collection. We thank the Director General of Health Malaysia for permission to publish this paper. This study was supported by the Malaria Research Centre at UNIMAS, a Fulbright Research scholarship through the US Department of State and funds from the Medical Research Council (MRC) UK (Grant number G0801971).

\section{Disclosures}

SK is working with QuantuMDx to develop improved diagnostics for malaria and is a shareholder (see nanomal.org for more information).

\section{Author details}

'Malaria Research Centre, Universiti Malaysia Sarawak, Kuching, Sarawak, Malaysia. ${ }^{2}$ Stanford University, Stanford, USA. ${ }^{3}$ University of St. Andrews, Scotland, UK. ${ }^{4}$ Institute for Infection and Immunity, St. George's, University of London, Cranmer Terrace, London SW17 ORE, UK. ${ }^{5}$ Sarikei Hospital, Sarikei, Sarawak, Malaysia.

Received: 6 December 2013 Accepted: 14 February 2014

Published: 18 February 2014

\section{References}

1. Singh B, Daneshvar C: Human infections and detection of Plasmodium knowlesi. Clin Microbiol Rev 2013, 26:165-184.

2. Tyagi RK, Das MK, Singh SS, Sharma YD: Discordance in drug resistanceassociated mutation patterns in marker genes of Plasmodium falciparum and Plasmodium knowlesi during coinfections. J Antimicrob Chemother 2013, 68:1081-1088

3. Cox-Singh J, Davis TME, Lee K-S, Shamsul SSG, Matusop A, Ratnam S, Rahman HA, Conway DJ, Singh B: Plasmodium knowlesi malaria in humans is widely distributed and potentially life-threatening. Clin Infect Dis 2008, 46:165-171.

4. Cox-Singh J, Hiu J, Lucas SB, Divis PC, Zulkarnaen M, Chandran P, Wong KT Adem P, Zaki SR, Singh B, Krishna S: Severe malaria - a case of fatal Plasmodium knowlesi infection with post-mortem findings: a case report. Malar J 2010, 9:10.

5. Daneshvar C, Davis TME, Cox-Singh J, Rafa'ee MZ, Zakaria SK, Divis PCS, Singh B: Clinical and laboratory features of human Plasmodium knowlesi infection. Clin Infect Dis 2009, 49:852-860.

6. Joveen-Neoh WF, Chong KL, Wong CMVL, Lau TY: Incidence of malaria in the Interior Division of Sabah, Malaysian Borneo, based on nested PCR J Parasitol Res 2011. doi:10.1155/2011/104284.

7. Barber BE, William T, Grigg MJ, Piera K, Yeo TW, Anstey NM: Evaluation of the sensitivity of a pLDH-based and an aldolase-based rapid diagnostic test for diagnosis of uncomplicated and severe malaria caused by PCR-confirmed Plasmodium knowlesi, Plasmodium falciparum, and Plasmodium vivax. J Clin Microbiol 2013, 51:1118-1123.

8. Barber BE, William T, Dhararaj P, Anderios F, Grigg MJ, Yeo TW, Anstey NM: Epidemiology of Plasmodium knowlesi malaria in north-east Sabah, Malaysia: family clusters and wide age distribution. Malar J 2012, 11:401.

9. Rajahram GS, Barber BE, William T, Menon J, Anstey NM, Yeo TW: Deaths due to Plasmodium knowlesi malaria in Sabah, Malaysia: association with reporting as Plasmodium malariae and delayed parenteral artesunate. Malar J 2012, 11:284

10. Barber BE, William T, Grigg MJ, Yeo TW, Anstey NM: Limitations of microscopy to differentiate Plasmodium species in a region co-endemic for Plasmodium falciparum, Plasmodium vivax and Plasmodium knowlesi. Malar J 2013, 12:8

11. Singh B, Kim Sung L, Matusop A, Radhakrishnan A, Shamsul SSG, Cox-Singh J, Thomas A, Conway DJ: A large focus of naturally acquired Plasmodium knowlesi infections in human beings. Lancet 2004, 363:1017-1024.

12. Lee K-S, Divis PCS, Zakaria SK, Matusop A, Julin RA, Conway DJ, Cox-Singh J, Singh B: Plasmodium knowlesi: reservoir hosts and tracking the emergence in humans and macaques. PLoS Pathog 2011, 7:e1002015.

13. Makler MT, Piper RC, Milhous WK: Lactate dehydrogenase and the diagnosis of malaria. Parasitol Today 1998, 14:376-377.

14. Moody A, Hunt-Cooke A, Gabbett E, Chiodini P: Performance of the OptiMAL malaria antigen capture dipstick for malaria diagnosis and treatment monitoring at the Hospital for Tropical Diseases, London. Br J Haematol 2000, 109:891-894.

15. Wongsrichanalai C, Arevalo I, Laoboonchai A, Yingyuen K, Miller RS, Magill AJ, Forney JR, Gasser RA Jr: Rapid diagnostic devices for malaria: field evaluation of a new prototype immunochromatographic assay for the detection of Plasmodium falciparum and non-falciparum Plasmodium. Am J Trop Med Hyg 2003, 69:26-30.

16. Murray CK, Gasser RA Jr, Magill AJ, Miller RS: Update on rapid diagnostic testing for malaria. Clin Microbiol Rev 2008, 21:97-110.

17. Chandrakanth G: Comparison of conventional blood film method and quantitative buffy coat and parasite lactate dehydrogenase, histidine rich protein-2. The rapid diagnosis of malaria. In Dissertation. Karnataka, Bangalore: Rajiv Gandhi University of Health Sciences; 2006 [http:// 14.139.159.4:8080/jspui/bitstream/123456789/1001/1/CDMMICR00022.pdf].

18. Thang ND, Erhart A, Hung LX, Thuan LK, Xa NX, Thanh NN, Ky PV, Coosemans M, Speybroeck N, D'Alessandro U: Rapid decrease of malaria morbidity following the introduction of community-based monitoring in a rural area of central Vietnam. Malar J 2009, 8:3.

19. McCutchan TF, Piper RC, Makler MT: Use of malaria rapid diagnostic test to identify Plasmodium knowlesi infection. Emerg Infect Dis 2008, 14:1750-1752.

20. Van Hellemond JJ, Rutten M, Koelewijn R, Zeeman AM, Verweij JJ, Wismans PJ, Kocken $\mathrm{CH}$, van Genderen PJJ: Human Plasmodium knowlesi infection detected by rapid diagnostic tests for malaria. Emerg Infect Dis 2009 , 15:1478-1480

21. Kawai S, Hirai M, Haruki K, Tanabe K, Chigusa Y: Cross-reactivity in rapid diagnostic tests between human malaria and zoonotic simian malaria parasite Plasmodium knowlesi infections. Parasitol Int 2009, 58:300-302.

22. Ong CWM, Lee SY, Koh WH, Ooi E-E, Tambyah PA: Monkey malaria in humans: a diagnostic dilemma with conflicting laboratory data. Am J Trop Med Hyg 2009, 80:927-928.

23. Berry A, Iriart X, Wilhelm N, Valentin A, Cassaing S, Witkowski B, Benoit-Vical F, Menard S, Olagnier D, Fillaux J, Sire S, Coustumier AL, Magnaval J-F: Imported Plasmodium knowlesi malaria in a French tourist returning from Thailand. Am J Trop Med Hyg 2011, 84:535-538.

24. Link L, Bart A, Verhaar N, van Gool T, Pronk M, Scharnhorst V: Molecular Detection of Plasmodium knowlesi in a Dutch Traveler by Real-Time PCR. J Clin Microbiol 2012, 50:2523-2524.

25. Bronner U, Divis PCS, Färnert A, Singh B: Swedish traveller with Plasmodium knowlesi malaria after visiting Malaysian Borneo. Malar J 2009, 8:15.

26. Cox-Singh J, Mahayet S, Abdullah MS, Singh B: Increased sensitivity of malaria detection by nested polymerase chain reaction using simple sampling and DNA extraction. Int J Parasito/ 1997, 27:1575-1577.

27. Singh B, Cox-Singh J, Miller AO, Abdullah MS, Snounou G, Rahman HA: Detection of malaria in Malaysia by nested polymerase chain reaction amplification of dried blood spots on filter papers. Trans $R$ Soc Trop Med Hyg 1996, 90:519-521.

28. Hackshaw A: Statistical Formulae for Calculating Some 95\% Confidence Intervals. In A Concise Guide to Clinical Trials. West Sussex, UK: WileyBlackwell; 2009:205-207.

29. Paramax-3 Rapid Test for Malaria. India: Zephyr Biomedicals; 2013 [http:// www.tulipgroup.com/Zephyr_New/qrg/paramax-3.pdf].

30. Bell D, Peeling RW: Evaluation of rapid diagnostic tests: malaria. Nat Rev Microbiol 2006, 4:S34-S38.

31. Hopkins H, González IJ, Polley SD, Angutoko P, Ategeka J, Asiimwe C, Agaba B, Kyabayinze DJ, Sutherland CJ, Perkins MD, Bell D: Highly sensitive detection of malaria parasitemia in a malaria-endemic setting: performance of a new loop-mediated isothermal amplification kit in a remote clinic in Uganda. J Infect Dis 2013, 208:645-652.

32. Lau Y-L, Fong M-Y, Mahmud R, Chang P-Y, Palaeya V, Cheong F-W, Chin L-C, Anthony CN, Al-Mekhlafi AM, Chen Y: Specific, sensitive and rapid detection of human Plasmodium knowlesi infection by loop-mediated isothermal amplification (LAMP) in blood samples. Malar J 2011, 10:197.

doi:10.1186/1475-2875-13-60

Cite this article as: Foster et al:: Evaluation of three rapid diagnostic tests for the detection of human infections with Plasmodium knowlesi. Malaria Journal 2014 13:60. 